\title{
Procedural Revision to the AOAC Germicidal Spray Products as Disinfectants Test Method: Establishment of Minimum and Maximum Log Density Values for Test Microbes on Inoculated Carriers
}

\author{
Rebecca M. Pines, Stephen F. Tomasino, ${ }^{1}$ and Michele P. Cottrill \\ U.S. Environmental Protection Agency, Office of Pesticide Programs, Microbiology Laboratory Branch, Environmental Science \\ Center, Fort Meade, MD 20755-5350 \\ Gordon C. HamiLton \\ Big Sky Statistical Analysts, Bozeman, MT 59715 \\ Albert E. Parker \\ Center for Biofilm Engineering, Montana State University, Bozeman, MT 59715
}

The AOAC Germicidal Spray Products as Disinfectants test method (AOAC Official Method 961.02) is used to measure the efficacy of spray products on hard inanimate surfaces; however, the method does not provide procedures to determine the population of the test microbe on inoculated glass slide carriers (e.g., carrier counts reported as CFU/carrier). Without a method to measure and monitor carrier counts, the associated efficacy data may not be reliable and repeatable. This report provides a standardized procedure to address this issue and, based on carrier count data collected by four laboratories from 2000 to 2010 , proposes a specific range for the mean log density per carrier as a requirement. Laboratory-based carrier count data were collected concurrently with 116 Method 961.02 efficacy tests conducted on spray products bearing claims against Pseudomonas aeruginosa and Staphylococcus aureus. For many of the tests a soil load (SL) was added to the inoculum (as specified on the product label claim). Six carriers were assayed per test for a total of 696 carriers. All but two of the $\mathbf{1 1 6}$ mean log densities were at least 5.0 (a geometric mean of $1.0 \times 10^{5} \mathrm{CFU/carrier).}$ Across the four combinations of microbes and SL treatments, the mean TestLD (mean log density across all enumerated carriers in a test) ranged from approximately 6.0 (a geometric mean of $0.9 \times 10^{6} \mathrm{CFU} /$ carrier) to 6.3 (a geometric mean of $2.0 \times 10^{6} \mathrm{CFU} /$ carrier). Across all microbes and SL treatments, the mean log density ( \pm SEM) was $6.2( \pm 0.07)$ per carrier (a geometric mean of $1.5 \times 10^{6} \mathrm{CFU} /$ carrier). The mean log density for six carriers per test showed good repeatability (0.32) and reproducibility (0.34). The proposed requirement for $S$. aureus tests and $P$. aeruginosa tests is a

\footnotetext{
Received October 26, 2012. Accepted by AH December 17, 2012.

1 Corresponding author's e-mail: Tomasino.Stephen@epamail.epa.gov

Appendixes are available on the J. AOAC Int. website, http://aoac. publisher.ingentaconnect.com/content/aoac/jaoac.

DOI: $10.5740 /$ jaoacint.12-406
}

mean log density (across six carriers) between $\mathbf{5 . 0}$ and 6.5. A separate 2009 study at three laboratories was conducted to evaluate the persistence of $P$. aeruginosa, $S$. aureus, and Salmonella enterica on glass carriers. Based on the persistence data, a $2 \mathrm{~h}$ use period is proposed for using the inoculated carriers post drying. The persistence data set was also used to assess the carrier counts for S. enterica. The carrier counts were approximately one log lower for $S$. enterica compared to $S$. aureus and $P$. aeruginosa; a range of 4.0 to 5.5 logs is proposed as a requirement for $S$. enterica tests.

$T$ The AOAC Germicidal Spray Products as Disinfectants test method (AOAC Official Method 961.02) is accepted by the U.S. Environmental Protection Agency (EPA) to verify bactericidal claims for disinfectant spray products (e.g., trigger sprays and aerosols). EPA's Office of Pesticide Programs (OPP), Biological and Economic Analysis Division, Microbiology Laboratory Branch, Fort Meade, MD, has worked closely with AOAC INTERNATIONAL and the user community to enhance the method through the official AOAC editorial revision process. As a result, a revised version of the method was published in the AOAC Official Methods of Analysis in 2009 (1). The editorial revisions focused on increasing the clarity of the method and eliminating the extensive cross-referencing to other methods, thereby creating a stand-alone procedure. Although the editorial revisions should provide more consistent test results across laboratories, targeted procedural changes will further enhance the method.

Currently, the Germicidal Spray Products as Disinfectants Test (GSPT) method lacks a standard procedure for the enumeration of the test microbe on untreated, dried inoculated glass slide carriers (referred to in this paper as "carrier counts"), and associated validity requirements (i.e., minimum and maximum levels of the test microbe). Without a method to measure and monitor control carrier counts, the associated efficacy data may not be as repeatable within a laboratory or reproducible between laboratories. Thus, we are proposing a set of procedural revisions to address these issues, namely, the establishment of a procedure to enumerate bacteria from inoculated control 
Table 1. Number of tests conducted in each laboratory for each combination of microbe and $S L$ value

\begin{tabular}{|c|c|c|c|c|c|c|}
\hline \multirow[b]{3}{*}{ Lab } & \multicolumn{3}{|c|}{ Pseudomonas aeruginosa } & \multicolumn{3}{|c|}{ Staphylococcus aureus } \\
\hline & \multicolumn{2}{|c|}{ SL } & \multirow[b]{2}{*}{ Total } & \multicolumn{2}{|c|}{ SL } & \multirow[b]{2}{*}{ Total } \\
\hline & Absent & Present & & Absent & Present & \\
\hline 1 & 2 & 1 & 3 & 5 & 1 & 6 \\
\hline 2 & 6 & 6 & 12 & 6 & 6 & 12 \\
\hline 3 & 3 & 5 & 8 & 4 & 5 & 9 \\
\hline 4 & 20 & 12 & 32 & 20 & 14 & 34 \\
\hline Total & 31 & 24 & 55 & 35 & 26 & 61 \\
\hline
\end{tabular}

carriers, a minimum and maximum control carrier count range to qualify the efficacy results, and a use period for inoculated carriers. A precedent for such changes was set in 2009 , when a similar set of procedural modifications was approved for the use-dilution method (2). Establishing minimum and maximum carrier counts, in conjunction with the published editorial revisions, will further standardize the GSPT and provide increased confidence in product efficacy results within and between laboratories. Most of the data presented in this report are associated with Staphylococcus aureus and Pseudomonas aeruginosa due to their importance in the EPA post-registration surveillance testing program designed to monitor the efficacy of disinfectant products. The present investigation uses an extensive, multilaboratory data set to support the proposed method modifications. In addition, a smaller data set associated with a microbe persistence study was used to establish a carrier count range for Salmonella enterica (subsp. enterica serovar Choleraesuis).

\section{Methods}

\section{Historical Data Collection}

The data were generated by four laboratories over an 11-year period (2000-2010) following the bacterial enumeration procedure described in this paper. Under the Antimicrobial Testing Program, OPP's Microbiology Laboratory Branch and three state Department of Agriculture laboratories (Michigan, Ohio, and North Carolina) collected the laboratory-based carrier count data concurrently while performing the efficacy evaluations. Product testing was conducted by trained analysts in accordance with EPA's Federal Insecticide, Fungicide, and Rodenticide Act Good Laboratory Practice Standards (3).

The laboratories strictly adhered to the published GSPT method for the stock culture initiation and maintenance, test culture preparation, and carrier inoculation steps. In all tests, no dilution of the final test cultures was performed. A total of 116 tests, distributed across the four laboratories, as shown in Table 1, were conducted on spray products bearing claims against $P$. aeruginosa and $S$. aureus with and without a soil load (SL) added to the inoculum (based on the product label). Nutrient broth was used as the growth medium for daily and final test cultures. Horse serum was used as the SL in most of the tests; fetal bovine serum was used in a few. SL was added at $5 \%(\mathrm{v} / \mathrm{v})$ to the inoculum before carrier inoculation. In each of the 116 tests, six carriers were analyzed per test day, for a total of 696 total carriers.

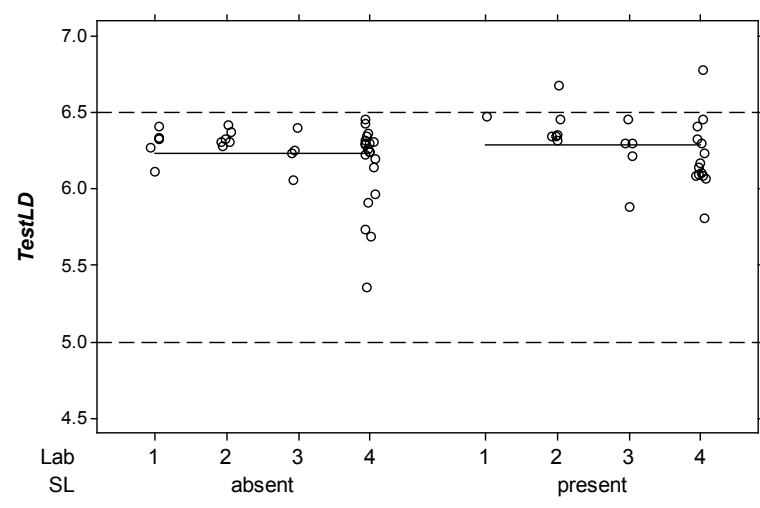

Figure 2. TestLD values for tests with $S$. aureus. Each point is a TestLD. Each solid line denotes the mean TestLD for the specified SL level. The dashed lines are at 5.0 and 6.5 , the proposed limits of validity for TestLD.

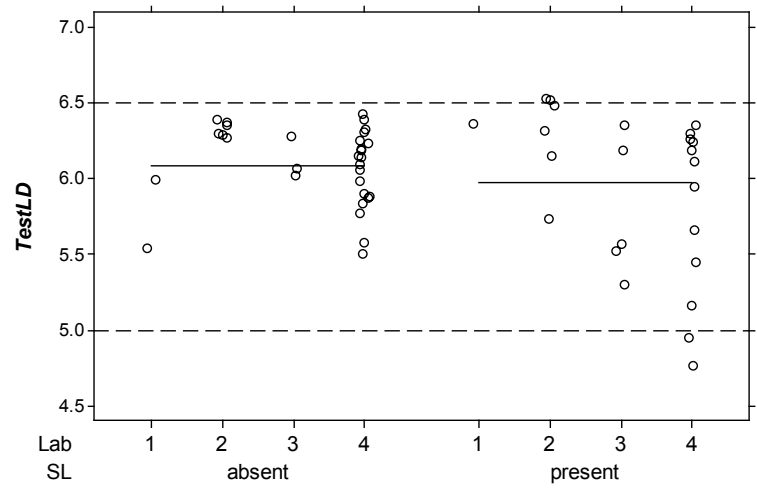

Figure 1. Test $L D$ values for tests with $P$. aeruginosa. Each point is a TestLD. Each solid line denotes the mean TestLD for the specified SL level. The dashed lines are at 5.0 and 6.5 , the proposed limits of validity for TestLD.

The carriers were randomly removed from the inoculated set after the drying step $\left(36 \pm 1^{\circ} \mathrm{C}\right.$ for $\left.40 \pm 2 \mathrm{~min}\right)$. Processing of carriers after drying was conducted within $2 \mathrm{~h}$ of drying. Control carrier counts, expressed as CFU/carrier, were entered into a Microsoft Access database; the data were sorted by laboratory, organism, date, and presence/absence of SL. The data were peer-reviewed by EPA laboratory staff and verified by the Quality Assurance Unit before statistical analysis. If an unusual observation was discovered during the peer review or statistical analysis process, the data were discussed with the appropriate laboratory personnel and necessary adjustments made. For one $P$. aeruginosa test, it was determined that the cell density for one of the six carriers was unusually large, and was the result of an error. That test was deleted from the data set before analysis and is not numbered among the 116 tests. No other adjustment to the submitted data set was made. Data for P. aeruginosa are provided in Appendix 1 (see Supplemental Material on J. AOAC Int. website, http://aoac.publisher.ingentaconnect. com/content/aoac/jaoac for Appendix 1-5), data for $S$. aureus in Appendix 2.

\section{Microbe Persistence on Glass Slide Carriers}

To augment the carrier count data, a study was conducted by the same three state laboratories over several months in 2009 
Table 2. Summary of the TestLD values (mean log densities, averaged across six carriers per test) for each microbe and SL combination

\begin{tabular}{lcccccc}
\hline Microbe & SL & $\begin{array}{c}\text { Mean } \\
\text { TestLD }\end{array}$ & SEM & $\mathrm{S}_{\mathrm{r}}$ & $\mathrm{S}_{\mathrm{R}}$ & $\begin{array}{c}\text { Geometric } \\
\text { mean of TestLD }\end{array}$ \\
\hline P. aeruginosa & Absent & 6.09 & 0.10 & 0.231 & 0.291 & $1.22 \times 10^{6}$ \\
& Present & 5.97 & 0.15 & 0.492 & 0.534 & $9.37 \times 10^{5}$ \\
S. aureus & Absent & 6.23 & 0.05 & 0.227 & 0.234 & $1.70 \times 10^{6}$ \\
& Present & 6.29 & 0.06 & 0.208 & 0.222 & $1.96 \times 10^{6}$ \\
Overall & & 6.17 & 0.07 & 0.321 & 0.342 & $1.47 \times 10^{6}$ \\
\hline
\end{tabular}

to evaluate the persistence of $P$. aeruginosa, $S$. aureus, and $S$. enterica on glass slide carriers over a $6 \mathrm{~h}$ period at room temperature using the same carrier count procedure. On each test day, the laboratories enumerated the viable inoculum on carriers with and without a 5\% (v/v) SL in a side-by-side fashion over four time periods post drying (15, 120, 240, and $360 \mathrm{~min}$ ). Three replicate tests were performed for each organism/SL/dry time combination with four carriers evaluated per treatment. At one of the three laboratories, the tests with a SL were performed 1 to 2 days after the corresponding experiment without a SL, except in one instance (for $S$. aureus) where there was a 7-day gap. Data for $P$. aeruginosa are provided in Appendix 3, data for S. aureus in Appendix 4, and data for S. enterica in Appendix 5.

\section{Statistical Analysis}

On each carrier, the density of viable bacteria (CFU/carrier) was measured and $\log _{10}$-transformed to form the $\log$ density (LD). The term TestLD is used to denote the mean LD across all enumerated carriers in a test. All statistical calculations were performed on the carrier LD values.

The historical data, over 11 years, were partitioned into four subsets, one for each combination of microbe and SL. An analysis of variance (ANOVA) was fit to each subset with random effects due to lab and "tests nested within lab." This model provided estimates of the reproducibility $\mathrm{SD}\left(\mathrm{S}_{\mathrm{R}}\right)$, which measures total variability among the four laboratories of the Test $L D$, and the repeatability SD $\left(\mathrm{S}_{\mathrm{r}}\right)$, which measures the within-laboratory variability of the Test $L D$. The test for a linear time trend of Test $L D$ values was conducted for each combination of microbe and SL by pooling the slopes across the four laboratories using an analysis of covariance (ANCOVA) with lab as a random effect and time as a covariate. Because there were four laboratories in the study, all reported $P$-values were calculated using three degrees of freedom.

The data from the persistence study in 2009 were partitioned into 24 subsets corresponding to the 24 combinations of microbe, time, and SL. For each of these subsets, an ANOVA model was fit, with lab and "tests nested within lab" as random effects. If all counts at the plated dilutions were zero, then a substitution rule of 0.5 was used at the lowest dilution for only one of the plates. This study was conducted using four carriers per test. In order to compare the results of these four-carrier tests with the historical data analysis presented in this report (which used six carriers per test), the $S_{r}$ and the $S_{R}$ were calculated from the formula for six carriers per test. To assess the effect of time on each of the six combinations of microbe and SL, an ANCOVA

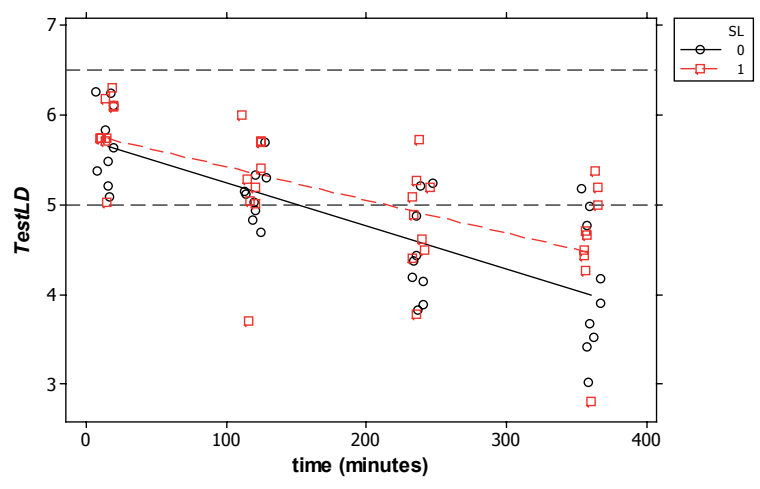

Figure 3. Persistence of $P$. aeruginosa on glass slide carriers. Each point is a TestLD for a specific SL. Although sampling only occurred at $15,120,240$, and $360 \mathrm{~min}$, the data have been jittered in the horizontal direction for ease in viewing. The regression lines indicate the decreasing trend over time for each SL level. The horizontal dashed lines are at 5.0 and 6.5 , the proposed limits of validity for TestLD.

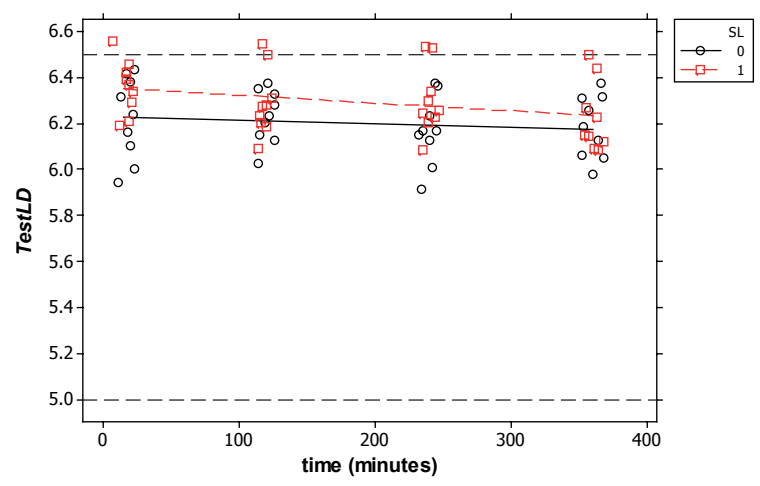

Figure 4. Persistence of Staphylococcus aureus on glass slide carriers. Each point is a TestLD for a specific SL. Although sampling only occurred at 15, 120, 240, and $360 \mathrm{~min}$, the data have been jittered in the horizontal direction for ease in viewing. The regression lines indicate the decreasing trend over time. The horizontal dashed lines are at 5.0 and 6.5 , the proposed limits of validity for TestLD.

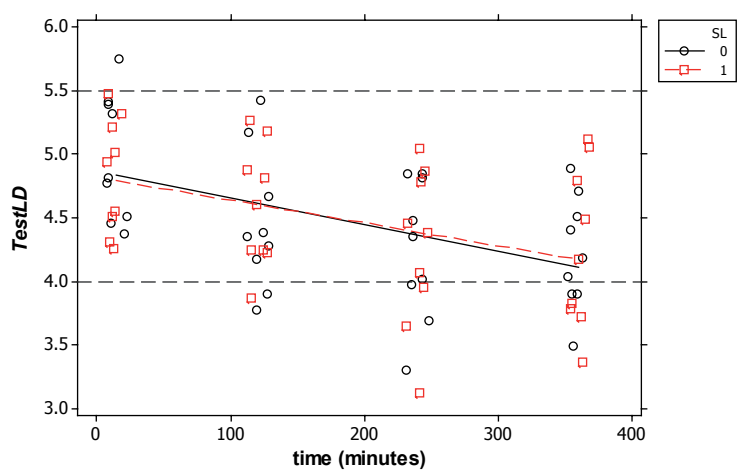

Figure 5. Persistence of $S$. enterica on glass slide carriers. Each point is a TestLD for a specific SL. Although sampling only occurred at $15,120,240$, and $360 \mathrm{~min}$, the data have been jittered in the horizontal direction for ease in viewing. The regression lines indicate the decreasing trend over time. The horizontal dashed lines are at 4.0 and 5.5 , the proposed limits of validity for TestLD. 
Table 3. Summary of the variability results associated with microbe persistence on glass slide carriers ${ }^{a}$

\begin{tabular}{|c|c|c|c|c|c|c|c|}
\hline \multirow[b]{2}{*}{ Organism } & \multirow[b]{2}{*}{ Time } & \multicolumn{3}{|c|}{ Without SL } & \multicolumn{3}{|c|}{ With $5 \%$ SL } \\
\hline & & Mean TestLD & $\mathrm{S}_{\mathrm{r}}$ & $S_{R}$ & Mean TestLD & $\mathrm{S}_{\mathrm{r}}$ & $S_{R}$ \\
\hline \multirow[t]{4}{*}{ S. aureus } & 15 & 6.22 & 0.1489 & 0.1859 & 6.36 & 0.0957 & 0.1213 \\
\hline & 120 & 6.23 & 0.1050 & 0.1157 & 6.29 & 0.1285 & 0.1465 \\
\hline & 240 & 6.17 & 0.1217 & 0.1527 & 6.30 & 0.1463 & 0.1463 \\
\hline & 360 & 6.18 & 0.1368 & 0.1368 & 6.22 & 0.1480 & 0.1480 \\
\hline \multirow[t]{4}{*}{ P. aeruginosa } & 15 & 5.69 & 0.2460 & 0.4854 & 5.84 & 0.3079 & 0.3880 \\
\hline & 120 & 5.12 & 0.2395 & 0.3031 & 5.22 & 0.5050 & 0.6935 \\
\hline & 240 & 4.47 & 0.1417 & 0.5963 & 4.83 & 0.4519 & 0.5971 \\
\hline & 360 & 4.07 & 0.3138 & 0.8338 & 4.55 & 0.6180 & 0.7816 \\
\hline \multirow[t]{4}{*}{ S. enterica } & 15 & 4.98 & 0.3302 & 0.5368 & 4.84 & 0.3336 & 0.4755 \\
\hline & 120 & 4.46 & 0.3548 & 0.5763 & 4.59 & 0.3011 & 0.5044 \\
\hline & 240 & 4.26 & 0.2225 & 0.6156 & 4.26 & 0.5980 & 0.5980 \\
\hline & 360 & 4.22 & 0.2970 & 0.4573 & 4.26 & 0.3439 & 0.7021 \\
\hline
\end{tabular}

although the actual tests were conducted with four carriers, these calculations show the predicted $\mathrm{S}_{\mathrm{r}}$ and the predicted $\mathrm{S}_{\mathrm{R}}$ for six carrier tests.

was fit, with lab and "tests nested within lab" as random effects and time as a covariate. Because experiments with and without SL were run side-by-side, SL differences in time trend were analyzed by first subtracting the paired SL responses from each other. The ANCOVA was fit to these differences. Because there were only three laboratories in the study, all reported $P$-values were calculated using 2 degrees of freedom.

For all data analyses, individual value plots, residual plots, normal probability plots, and the Anderson-Darling test for normality (4) were used to identify unusual data and to assess model fit. All ANOVA and ANCOVA models were implemented using the statistical software $\mathrm{R}$, package nlme $(5,6)$. All statements of statistical significance are made with respect to a significance level of $5 \%$.

\section{Results and Discussion}

\section{Historical Data}

The LD values for $S$. aureus and $P$. aeruginosa are plotted in Figures 1 and 2, and the summaries of the data are presented in Table 2. Across the four combinations of microbes and SL treatments, the mean Test $L D$ ranged from approximately 6.0 (a geometric mean density of $0.9 \times 10^{6} \mathrm{CFU} /$ carrier) to 6.3 (a geometric mean density of $2.0 \times 10^{6} \mathrm{CFU} /$ carrier). All but two of the 116 Test $L D$ values were at least 5.0 (a geometric mean of $1.0 \times 10^{5} \mathrm{CFU} /$ carrier). The maximum observed Test $L D$ was 6.53 among $P$. aeruginosa tests and 6.77 among $S$. aureus tests. The Test $L D$ values per microbe and SL combination showed good repeatability (range of 0.208 to 0.492 ) and reproducibility (range of 0.222 to 0.534 ). Across microbes and SL treatments, the mean Test $L D( \pm$ SEM, standard error of the mean) was $6.2( \pm 0.07)$ (a geometric mean of $1.5 \times 10^{6} \mathrm{CFU} /$ carrier). The data presented here form the basis for the minimum and maximum carrier count.

Plots of Test $L D$ s against date and experiment number (data not shown) did not produce an obvious time trend. The ANCOVA produced a small, not statistically significant $(P \geq 0.07)$, positive linear slope. The homogeneity of variances assumption was not discredited by the data; for each of the four combinations of microbe and SL, the within-laboratory variances of TestLDs were not statistically significantly different among the laboratories. The normality assumption was discredited by the data; the Anderson-Darling test for normality for the residuals of the ANOVA model showed statistically significant deviations from normality due to an occasional unusually small LD value. We believe that those few unusual values represent the inherent variability of the GSPT, and that the number of experiments is sufficiently large that the statistical comparison of means is reliable even though the data do not conform to the normality assumption. Based on the analysis of the historical data set, we propose a minimum Test $L D$ of 5.0 (a geometric mean of $1.0 \times 10^{5} \mathrm{CFU} /$ carrier) and a maximum TestLD of 6.5 (a geometric mean of $3.2 \times 10^{6} \mathrm{CFU} /$ carrier) as a requirement for $S$. aureus tests and P. aeruginosa tests. This range provides for the potential inherent variability that may be experienced across a wide range of laboratories and the slight effect due to the addition of an SL. Thus, any tests producing a mean log density lower than 5.0 or greater than 6.5 would be invalidated and would have to be repeated, except for two retesting scenarios (see description below).

In order to reduce the potential of acquiring carrier counts higher than the upper limit, additional steps are recommended to allow for dilution (using sterile broth) of the final 48-54 h test culture. Conversely, over-dilution of the final test culture may result in carrier counts lower than the $5 \log$ minimum. To adjust the microbial titer, the harvested inoculum may be diluted in sterile broth before the addition of a SL (if required). Although unlikely, concentration of the final test cultures may be necessary to increase the bacterial titer before carrier inoculation. Guidance for dilution of the test culture is provided in the proposed text for the method revision as presented below. Based on the product's efficacy, repeat testing scenarios when carrier counts fall above or below the established range must be addressed as revisions to the method, as well. 


\section{Persistence Data}

The mean Test $L D$ values are plotted as a function of time for each of $P$. aeruginosa, $S$. aureus, and $S$. enterica in Figures 3-5, respectively. Each figure depicts the linear trend for the Test $L D$ s both with and without SL. When the SL levels were analyzed separately, only $S$. enterica, both with and without SL, exhibited a statistically significant decrease in the mean $\log$ density over time $(P$-value $<0.05$ for $S$. enterica; $P$-value $<0.10$ for $P$. aeruginosa; $P$-value $>0.05$ for $S$. aureus). However, when pooled across both SL levels, all three microbes exhibited significant negative trends $(P$-value $<0.05)$. Pooling was appropriate since none of the microbes exhibited statistically significantly different trends between SLs ( $P$-value $\geq 0.07$ ). Per hour, the overall decreasing linear trend in the mean $\log$ density was 0.25 for P. aeruginosa, 0.02 for $S$. aureus, and 0.12 for $S$. enterica.

For each microbe and point in time, the mean TestLD, $\mathrm{S}_{\mathrm{r}}$, and $\mathrm{S}_{\mathrm{R}}$ are provided in Table 3. After $2 \mathrm{~h}$, there was a noticeable drop in the mean TestLD for P. aeruginosa and S. enterica. At $6 \mathrm{~h}$, there was a marked increase in the $\mathrm{S}_{\mathrm{R}}$ for $P$. aeruginosa both with and without a SL, and an increase in the $\mathrm{S}_{\mathrm{R}}$ for $S$. enterica with a SL.

Based on these persistence study data, a minimum TestLD (calculated from six carriers) of 4.0 (a geometric mean of $1.0 \times 10^{4} \mathrm{CFU} /$ carrier) and a maximum Test $L D$ (calculated from six carriers) of 5.5 (a geometric mean $3.2 \times 10^{5} \mathrm{CFU} /$ carrier) are recommended for carriers inoculated with $S$. enterica. Over all the data, 16 Test $L D$ s fell below 4.0 and one was larger than 5.5 (Figure 5). However, when only the first $2 \mathrm{~h}$ of data are considered, only three Test $L D$ s fell below 4.0.

The current GSPT indicates that carriers must be used on the day of preparation. This use period is too long, especially for $P$. aeruginosa and S. enterica, and should be revised. Based on the data generated during the persistence study, it is advisable to perform the efficacy testing and the carrier counts within $2 \mathrm{~h}$ after drying to mitigate a loss of viable inoculum.

AOAC Official Method $\mathbf{9 6 1 . 0 2}$ has been revised to include the procedural changes described in this report. The proposed carrier count procedure for the GSPT method is provided as follows:

Carrier Count Procedure for AOAC Official Method 961.02 (to follow section C. Operating Technique.-(i) Verification of positive carriers).

(j) Enumeration of viable bacteria from carriers (carrier counts).-After the carriers have dried, assay carriers in two sets of three carriers, one set prior to conducting the efficacy tests and one set following the test. Place each of the inoculated, dried carriers in a $38 \times 100 \mathrm{~mm}$ culture tube or sterile $50 \mathrm{~mL}$ polypropylene conical tube containing $20 \mathrm{~mL}$ letheen broth. Vortex immediately $-60 \pm 5 \mathrm{~s}$ for $P$. aeruginosa or $120 \pm 5 \mathrm{~s}$ for $S$. aureus and $S$. enterica. After vortexing, make serial 10 -fold dilutions in $9 \mathrm{~mL}$ phosphate-buffered dilution water. If the serial dilutions are not made and plated immediately, keep the vortexed tubes at $2-5^{\circ} \mathrm{C}$ until this step can be done. However, dilution and plating should be performed within $2 \mathrm{~h}$ of vortexing. Alternatively, the letheen broth tubes may be pooled after vortexing for each set of three carriers. An aliquot of the pooled media $(60 \mathrm{~mL})$ will be serially diluted and plated, and the average carrier count per set will be calculated.

Plate $0.1 \mathrm{~mL}$ aliquots of appropriate dilutions in duplicate on tryptic soy agar (TSA) or TSA with $5 \%$ sheep blood using pouror surface-spread plating; dilutions of $10^{-1}$ through $10^{-3}$ should result in plates with a countable range of colonies. Briefly mix each serial dilution tube on a vortex mixer before plating. For pour-plating, add molten TSA tempered to approximately $45^{\circ} \mathrm{C}$ to each plate. Swirl the pour-plates to distribute cells evenly and allow agar to solidify. Incubate plates (inverted) concurrently with the efficacy subculture tubes at $36 \pm 1^{\circ} \mathrm{C}$ for up to $48 \pm 2 \mathrm{~h}$. Count the colonies by hand or with a colony counter. Use dilutions yielding counts up to 300 for enumeration; plate counts of 0 are to be included in the calculations.

Calculate the $\log _{10}$ density (LD) for each carrier by taking the $\log _{10}$ of the density (per carrier). The mean LD across carriers is the Test $L D$ for the test. The Test $L D$ must be at least 5.0 (corresponding to a geometric mean density of $1.0 \times 10^{5}$ ) and not above 6.5 (corresponding to a geometric mean density of $3.2 \times 10^{6}$ ) for $P$. aeruginosa and $S$. aureus tests. A Test $L D$ below 5.0 or above 6.5 invalidates the test, except for two retesting scenarios (see Retesting Guidance below). For S. enterica, the Test $L D$ must be at least 4.0 (corresponding to a geometric mean density of $1.0 \times 10^{4}$ ) and not above 5.5 (corresponding to a geometric mean density of $3.2 \times 10^{5}$ ). A Test $L D$ below 4.0 or above 5.5 invalidates the test, except for two retesting scenarios (see Retesting Guidance below).

Note: If the GSPT method is strictly followed, TestLDs of at least 5.0 (for P. aeruginosa and $S$. aureus) and 4.0 (for $S$. enterica) are expected; values lower than these levels may indicate a dilution error, poor media quality, interference by environmental parameters (e.g., carrier drying and culture incubation conditions), or lack of adherence to the method. The prescribed minimum count also accounts for the addition of $5 \%$ SL to the inoculum.

Note: For the purpose of achieving the carrier count range, dilution of the final test culture may be performed using the sterile culture medium used to generate the final test culture (e.g., nutrient broth). Dilution of the final test culture (e.g., one part culture plus one part sterile broth) should be made before addition of the SL to the inoculum. Although unlikely, concentration of the final test culture may be necessary if the bacterial titer in the final test cultures is too low. Concentration may be achieved using centrifugation (e.g., $5000 \times g$ for $20 \mathrm{~min}$ ) and resuspending the pellet in the appropriate volume of the sterile final test culture medium necessary to meet the carrier count range. In addition, the use of a spectrophotometer to measure optical density (OD $650 \mathrm{~nm}$ ) is recommended to provide a tool (i.e., development of a standard curve) for assessing the need to concentrate or dilute the final test culture. Sterile broth medium should always be used to calibrate the spectrophotometer.

Retesting guidance.-For tests where the product passes and the Test $L D$ value is above 6.5 for $S$. aureus and P. aeruginosa or above 5.5 for $S$. enterica, no retesting is necessary. For a test where the product fails and the Test $L D$ is below 5.0 for $S$. aureus and P. aeruginosa or below 4.0 for $S$. enterica, no retesting is necessary. For tests where the product fails and the mean Test $L D$ is above 6.5 for $S$. aureus and P. aeruginosa or above 5.5 for $S$. enterica, retesting may be conducted. These changes are only for the organisms specified in this report.

Revision for AOAC Official Method 961.02, section C. Operating Technique.-(e) Carrier inoculation.-Delete the following statement: "Inoculated carriers must be used on 
day of preparation." Insert the following statements: "Note: Use inoculated carriers for determining carrier counts, (j), and performing efficacy testing. Use inoculated carriers within $2 \mathrm{~h}$ of drying."

\section{Acknowledgments}

The statistical analyses were performed under contract between the Center for Biofilm Engineering at Montana State University and the EPA, OPP, Biological and Economic Analysis Division, Microbiology Laboratory Branch. We thank Kiran Verma, Quality Assurance Officer for the EPA Microbiology Laboratory, for conducting the audit of data that appear in this report. We express our appreciation to the following Department of Agriculture Laboratories for their participation in the EPA Antimicrobial Testing Program and generation of the carrier count data: Michigan's William C. Geagley Laboratory, East Lansing, MI; Ohio's Consumer Analytical Laboratory, Reynoldsburg, $\mathrm{OH}$; and North Carolina's Constable Laboratory, Raleigh, NC.

\section{References}

(1) Official Methods of Analysis (2013) 18th Ed., AOAC INTERNATIONAL, Gaithersburg, MD, Method 961.02

(2) Tomasino, S.F., Pines, R.M., \& Hamilton, M.A. (2009) J. AOAC Int. 92, 1531-1540

(3) Code of Federal Regulations (2005) Part 160, Good Laboratory Practice Standards, U.S. Government Printing Office, Washington, DC

(4) Pomory, C.M. (2006) J. Appl. Stat. 33, 461-462. http://dx.doi. org/10.1080/02664760600677720

(5) R Development Core Team (2010) R: A Language and Environment for Statistical Computing, R Foundation for Statistical Computing, Vienna, Austria, ISBN 3-900051-07-0, URL http://www.R-project.org

(6) Pinheiro, J., Bates, D., DebRoy, S., Sarkar, D., \& The R Core Team (2009) nlme: Linear and Nonlinear Mixed Effects Models, R Package Version 3.1-96, R Foundation for Statistical Computing, Vienna, Austria 